And when it comes to food metabolomics studies, researchers often incorporate additional data, such as sensory information-ratings from a panel of experts who grade the products on such variables as color, mouth feel, fruitiness, and acidity, providing a metric against which to correlate the chemical data themselves.

Markus Herderich, research group manager at the Australian Wine Research Institute in Adelaide, uses these elements in his own research. Several years ago, Herderich's team set out to find the chemicals underlying the black-pepper aroma characterizing some Australian Shiraz wines. Given the question, the obvious first step would be to ask whether the compound that gives black pepper its flavor (as opposed to its "heat") is also found in wine. Unfortunately for Herderich, that compound had never been identified. That meant the team had no idea what to look for in the Shiraz.

It took 2 years to assemble a set of 18 Shiraz grape samples harvested in 2002 and 2003 from 12 vineyards representing different levels of pepper intensity. "We had the biological variability covered," Herderich says. Then there was another six months spent compiling sensory data quantifying "pepper" levels. After this the team subjected the samples to "headspace" GC-MS-basically, analyzing the volatile aromatic components of the grapes themselves-and crunched the numbers, looking for mass spectrometry features that spiked with the sensory readings.

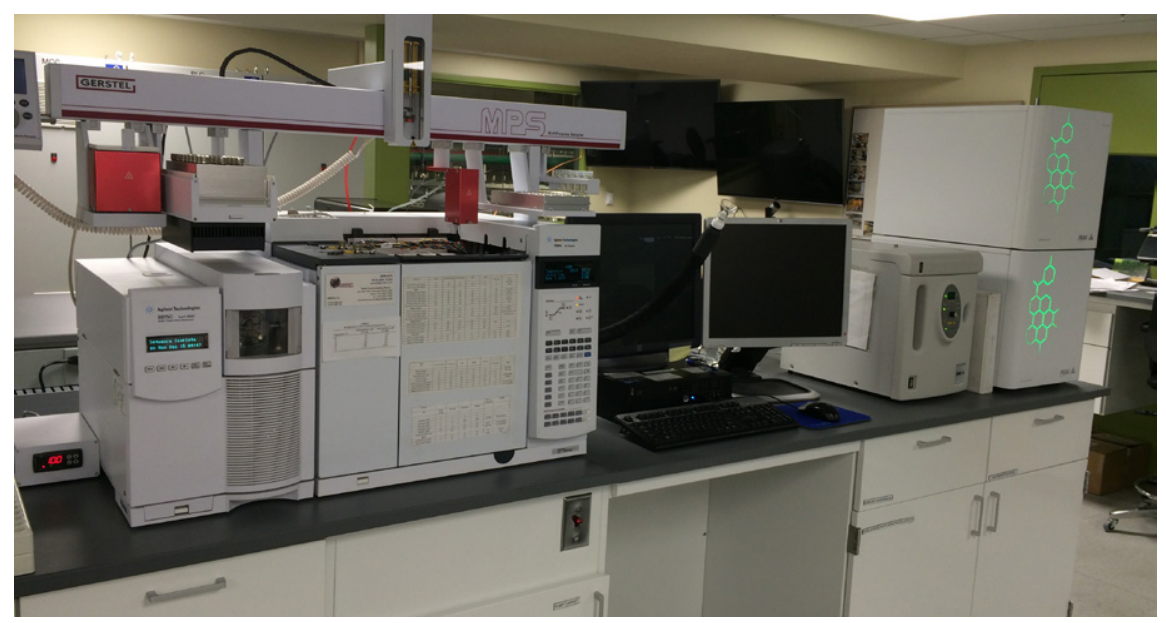

GC-MS with olfactory port in the analytical lab at New Belgium Brewery. Credit: Dana Sedin
Since they didn't know precisely what they were looking for, Herderich's team had to take an "untargeted" metabolomics approach-they let the mass spectrometer look at everything, then used analytical software and statistical methods to sift through the data. From all of these data and analyses, a sesquiterpene known as alphaylangene emerged. But when the team tried to establish alpha-ylangene as the chemical behind the peppery smell, they were stymied-while the compound was present in grapes, it was not found in wine. To top it off, it didn't smell like pepper-in fact, it didn't smell like anything. It seemed to be a proxy for "pepperiness," but not the source of the flavor. "There's a fine difference between a biomarker and a bioactive [compound]," Herderich explains.

The team went back to the drawing board. They enriched the odorant sufficiently from peppery grapes to begin compiling a chemical dossier. They used a specialized GC-MS outfitted with an "olfactory detector"-an outlet that looks a bit like a medical oxygen mask and allows users to smell compounds as they emerge from the column-to hone in on the peppery aroma during chromatographic separations. One smell bore a striking resemblance to the peppery aroma of pepper itself. Herderich and his team then set out to isolate this chemical from peppercorns, where it would be considerably more abundant.

Their labors yielded another sesquiterpene, this one called rotundone. It turns out that wines and grapes with

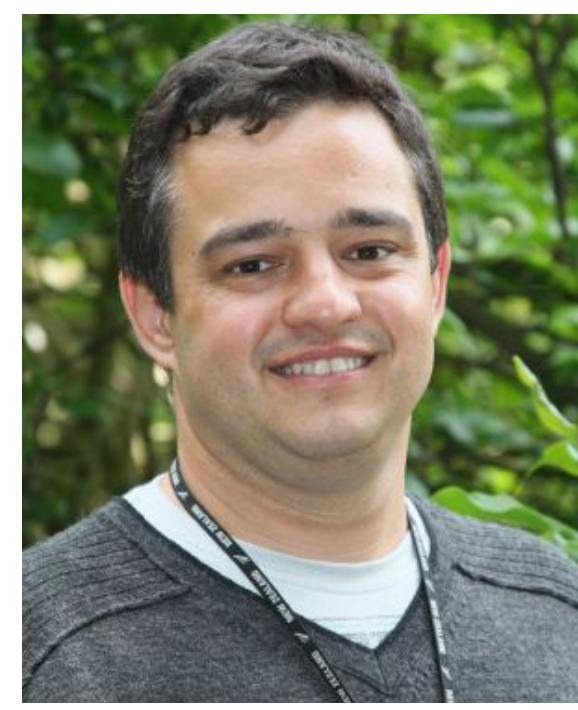

Silas Villas-Boas. Image courtesy of Dr. Villas-Boras.

strong peppery aromas had high rotundone levels; those with barely detectable pepperiness had little. And, adding isolated rotundone to water or wine was sufficient to impart a peppery aroma. Rotundone, the authors concluded, was the chemical putting the "pepper" in black pepper.

According to Herderich, his discovery allows wine makers to "tweak" the pepperiness of their wines by selecting grapes with more or less rotundone. It also explains a curious finding with the sensory panel. Once rotundone was identified, the researchers diluted the purified compound in water and wine at different concentrations, then asked their panelists if they could detect it. Most panelist were highly attuned to the chemical, picking up as little as 8 ng/L in water. But $20 \%$ couldn't detect it even at 500 times that concentration. As it turns out, Herderich says, "a quarter of the population is close to anosmic" for rotundone-they cannot perceive it-explaining how two people can experience the same wine differently, or have different preferences for peppery food. "It may simply reflect your personal ability to perceive that compound."

\section{Signs of aging}

Across the globe at Colorado State University (CSU) in Fort Collins, Jessica Prenni is also applying metabolomics to fermented beverages. But in this city rich with microbreweries, Prenni's interest lies not in wine, but beer. 
Prenni is director of the Proteomics and Metabolomics Facility at CSU, and her lab handles projects on topics ranging from infectious disease to animal breeding. More recently, though, they have been tackling food science.

Several years ago she decided to look at beers using LC-MS, just for fun. Most work on beer is done via GC-MS, as it's the volatile compounds consumers notice. "We were using [beer] as a sample type that we could use for educational purposes," Prenni explains, "so we could talk to people about our workflow without putting them to sleep."

Working with colleagues at New Belgium Brewery in Fort Collinsthe maker of Fat Tire beer-Prenni's lab separated and characterized nearly 7000 "molecular features" of freshly brewed beer and beers that had been stored either in the refrigerator or at room temperature for up to 16 weeks. Sixteen metabolites varied significantly among conditions, but the team zeroed in on a single compound,

5-methylthioadenosine (5-MTA), that had never been described in beer and whose abundance seemed to be correlated with age.

5-MTA, Prenni says, is essentially a biomarker of beer aging. "As beer ages, things happen to the beer, and the flavor changes," Prenni explains. "They [brewers] want to understand that process and what's happening, and figure out ways to slow it down."

At the moment, if brewers want to determine whether a change affects shelf life, the only way to find out is to put the bottles on the shelf, wait, and then ask a sensory panel to judge what happened. 5-MTA could serve as a chemical proxy, providing a quantifiable molecular indicator of aging-at least in New Belgium beers. Though 5-MTA has been observed in both the company's
Fat Tire and Ranger beers, it isn't yet clear how ubiquitous the marker is.

Dana Sedin, analytical lab manager at New Belgium Brewery, says the company still relies on sensory panels for its aging work, but it is starting to measure 5-MTA abundance. In one case, he says, his lab wanted to know what would happen to shelf-life if they changed hops. "The work that Jessica's team did showed that yes, one of the hops that we had selected did show an improvement in shelf life. And that happened to correlate with the sensory data we had."

Mattivi's team took 20 bottles each of 20 Sangiovese red wines and stored them either in a climate controlled wine cellar (below $18^{\circ} \mathrm{C}$ ) or at room temperature, as might be done in a house or restaurant. Using an untargeted LC-MS approach, the team identified more than 1000 nonvolatile compounds, most of which remained constant over time and temperature. Some, though, did notthe products of oxidation and hydroIysis reactions, which occur some three to four times faster at higher temperatures than at lower ones. Red pigments, for instance, degrade more rapidly at higher temperatures, while yellow/ brown pigments a c cumulate, imparting to the wine a duller, flatter color than when it was made. Mattivi says a wine stored 6 months at room temperature is equivalent to one aged 18 months in a wine cellar. "So, I should add one year to what is written on the label," he says.

Two metabolites did stand out, he says, the apparent result of

Another possible application is in screening. If a brewery were to test a dozen experimental formulations of beer, sensory panels would be overwhelmed, Sedin says. 5-MTA measurements could allow the brewers to narrow the possibilities before bringing in a sensory panel. "I look at it more as a future screening technique to allow us to run bigger trials," he says.

Aging is also a concern among oenophiles-though there, of course, age is considered a virtue, assuming the wine is stored correctly. Fulvio Mattivi, research manager of the Department of Food Quality and Nutrition at the Fondazione Edmund Mach in Italy, presented data at a recent American Chemical Society symposium describing putative metabolite markers for aging in wine. the reaction of sulfur dioxide, bisulfite (a preservative), and wine tannins: epicatechin 4-beta-sulfonate and procyanidin B2-4-beta-sulfonate, whose identities were confirmed by NMR. These compounds, he says, do not occur in properly stored wine, and their abundance increases the longer they are stored at elevated temperatures. As a result, they are biomarkers of improper storage-and potentially useful tools for wineries fielding complaints from customers dissatisfied when they open a bottle after long storage. Mattivi's recommendation: Unless you have a climate-controlled cellar, save your money, and try not to sit on your wines too long. "If you have a very good bottle of wine, and if you are waiting for a special occasion to open it ... drink it soon." 
TAKE ACTION AGAINST

\section{CONTAMINATION WITH \\ $\mathrm{QMI}^{\oplus}$ SAFE-SEPTUM}

\section{Sample, Inoculate Or Add Nutrients}

To Your Bioreactor Aseptically !

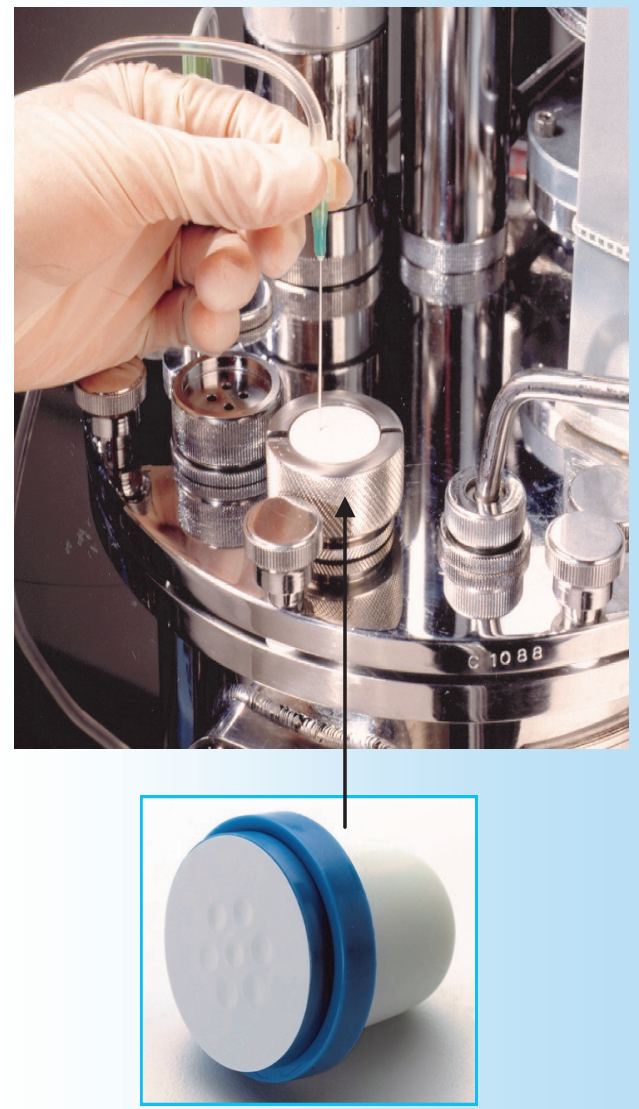

The QMI Safe-Septum is:

- Aseptic

- Pressure \& Temperature Safe

- Pre-Sterilized

- Easy To Retrofit

- Validated

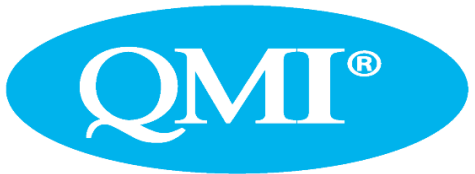

651-501-2337

Email: info@qmisystems.com
Cheese, Gromit!

You may want to pair that wine with cheese, another fermented product receiving metabolomics attention recently. Metabolite analysis of cheese isn't new-as with wine and beer, researchers have been studying the biology and chemistry of cheese-making for years. But, says Anne Thierry, senior researcher at the French National Institute for Agricultural Research (INRA) in Rennes, that previous work was often "targeted"-that is, focused on specific, known metabolites. "[Untargeted] metabolomics is a way to open new doors," she suggests.

Thierry is interested in how cheese changes as it ripens-information that then can be used to understand, for instance, how different microorganisms collaborate to create a finished camembert or emmental.

In 2013, Thierry and her then-graduate student Clementine Le Boucher, in collaboration with colleagues from the Atlantic College of Veterinary Medicine, Food Science and Engineering (Oniris) in Nantes, demonstrated for the first time that cheese is amenable to metabolomics analysis. Using a "model cheese"-essentially a concentrated milk product mixed with a single strain of lactic acid bacteria in the absence of fats-the team extracted water-soluble metabolites after 0,8 , and 48 hours of bacterial incubation and subjected these to LC-MS. They profiled volatiles by analyzing the headspace over the cheese using GC-MS.

In total, Thierry's team identified 45 metabolites whose abundance varied with time, 20 using LC-MS. Twelve were amino acids, but also present were B-vitamins-compounds that largely have been overlooked in cheesemaking circles for decades, as well as milk metabolites such as L-carnitine and creatine.

Now Thierry's team has moved on to more complex questions, such as how bacterial distribution influences ripening.
They incubated their model cheeses with the same number of bacteria, but distributed either in a small number of large colonies or numerous smaller ones. "Of course, the final cheeses were not so different, but from the beginning to the end of ripening we were able to identify significant differences between these two model cheeses," she says.

Whether those metabolites turn out to be important in cheese-making remains up in the air. Complex as it is, metabolomics, like other -omics disciplines, is merely a hypothesis-generating tool, a first step in a long workflow that sometimes yields actionable information. It is valuable, to be sure. But, don't look to find metabolomics laboratories in your favorite breweries, wineries, and fromageries - the expertise and hardware required are simply too expensive. It is more likely that companies will collaborate with outside experts as the need arises.

As for consumers, they can enjoy their wine, beer, and cheese blissfully unaware of the complex chemistry going on in the background.

Written by Jeffrey M. Perkel, Ph.D. [四

BioTechniques 58:8-12 (January 2015) doi 10.2144/000114244 